\title{
Optimum Designed Micro Active Forceps with Built-in Fiberscope for Retinal Microsurgery
}

\author{
Koji Ikuta' ${ }^{1}$ Takashi Kato ${ }^{1}$, and Satoru Nagata ${ }^{2}$ \\ ${ }^{1}$ Department of Micro System Engineering, School of Engineering, Nagoya University, \\ Furo-cho, Chikusa-ku, Nagoya 464-8603, Japan \\ ikuta@mech.nagoya-u.ac.jp \\ kato@bio.bmse.mech.nagoya-u.ac.jp \\ http://www.bmse.mech.nagoya-u.ac.jp \\ ${ }^{2}$ Department of Ophthalmology, Shiga University of Medical Science, Tsukiwa-cho, \\ Seta, Otsu, Shiga 520-2192, Japan \\ nagataesums.shiga-med.ac.jp \\ http://www.hitl.washington. edu/people/nagata
}

\begin{abstract}
A new concept of eye microsurgical system is proposed. The final goal of our research project is not only improving surgical instruments but also establishing a total surgical system applicable to today's most difficult microsurgery at the bottom of an eyeball. The new prototype of micro forceps, which has a joint to enlarge the surgical area and also has a built-in thin optical fiberscope to increase the dexterity of retinal microsurgery, was designed as the first step of a long range research plan. Since this micro active forceps is equipped with thin optical fiber inside the stem to obtain a lateral view of the retina, drastic improvement of fine operations can be achieved. And the design of active joint and end-effector were optimized theoretically.
\end{abstract}

\section{Introduction}

Microsurgery is the most attractive but most difficult surgical treatment at present. Many fields in medicine have been developing special tools along with special surgical skills. Nowadays, it is claimed there is a great demand for various kinds of microsurgery using micro system technology.

Demand for eye microsurgery is especially clear for the engineer to understand. Eye microsurgery on the retina needs extremely difficult and special skills. Related issues cover general aspects of all kinds of microsurgery. Therefore, technology developed for this subject could be easily generalized to other medical fields.

This paper reports a contribution from micro technology as the first step of a longterm and systematic research plan[1,2]. 


\section{Problems of Today's Eye Microsurgery on the Retina}

In this chapter, we would like to explain the representative method of conventional retinal microsurgery and also refer to problems resulting from the characteristics of instruments in use.

\subsection{Eye microsurgery at present}

Let's start with a brief introduction of current eye microsurgery. Fig. 1 shows today's microsurgery of an eyeball[3]. The retina and thin membrane on the eye ground are operated A highly trained skillful surgeon handles special forceps under a binocular microscope while a light guide is controlled to illuminate the small surgical area.

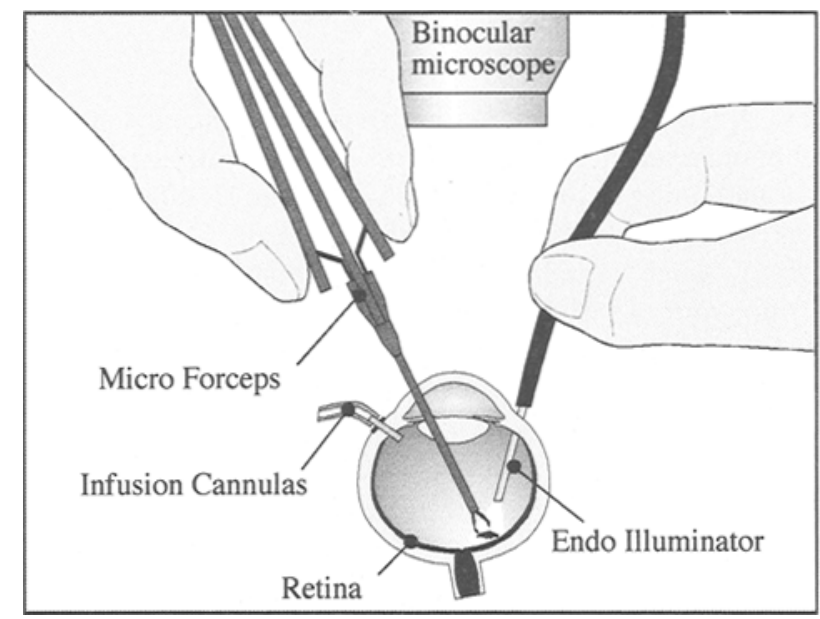

Fig. 1. Conventional eye microsurgery on the retina. A surgeon and an assistant work together looking at indirect retinal top view via binocular microscope. Illumination is also provided from microscope but it is not sufficient for the operation.

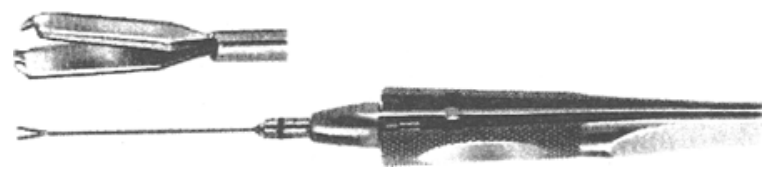

Fig. 2. Example of forceps-type conventional micro instrument for eye microsurgery. Length of the thin metal tube (neck) is about $30 \mathrm{~mm}$ and diameter of that is about $1 \mathrm{~mm}$. 


\subsection{Problems}

This surgical method and instrumentation entail two serious issues as follows.

\section{1) Limitation of operational approach}

The conventional forceps is the straight bar type as shown in Fig. 2 which is maneuvered via a small hole which acts as a pivot on the upper half of the sclera. Thus the working space of the tip of micro forceps is quite limited (only the bottom half of eyeball can be reached). The situation above is explained by Fig. 3 .

\section{2) Limitation of visual information}

Only a top view image through the cornea by a binocular microscope is available. It is extremely difficult to grasp the depth and the distance information between the forceps' tip and the retina. Sometimes the retina has been seriously damaged by young doctors' mistakes.

\section{Approach Concepts}

Before describing technological articles, we would like to describe our concept of research approach and the development policy.

Our final goal is not only improving surgical tools but also establishing a new microsurgical system applicable to today's most difficult microsurgery. In this kind of research, people on the engineering side should be devote great attention to the interface between the new technology and its users. It is needless to say that the users here mean medical doctors.

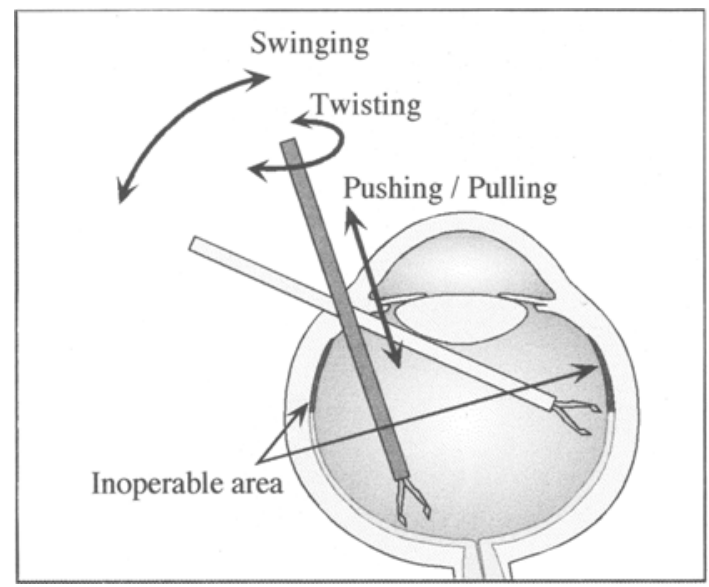

Fig. 3. Limitation of operational area by conventional forceps. The hole on the cornea acts as a pivot of rotational motion. 
Unfortunately, there has been much misunderstanding of developments in the medical engineering field. Even though the technology itself may be excellent, it would never be used if medical doctors were displeased with it. It is obvious that the main cause of above unhappy cases lies on the miscommunication between engineers and doctors.

Another big reason is conservatism in medicine. Most surgeons like to keep their own skills cultivated through long periods of clinical training. It is extremely difficult to break out of this closed situation. One solution, the authors think, would be $a$ drastic improvement of instrumentation while preserving the continuity of operational skills.

Based on above considerations, we decided to start with the improvement of tools such as micro forceps as the first step. The second step will be the total system as shown in Fig. 11, which will be described later.

\section{Micro Active Forceps}

The mechanisms and handling methods of micro active forceps made for trials in our laboratory are described in this chapter.

The term "active" used here does not mean that electrical devices, i.e. actuators, control each mechanism, but that positive approaches to and observations of the diseased part are possible with articulated forceps with a fiberscope. It is based on the belief that the continuity between the surgeon's skill and surgical instruments should be given particular consideration in microsurgical technologies.

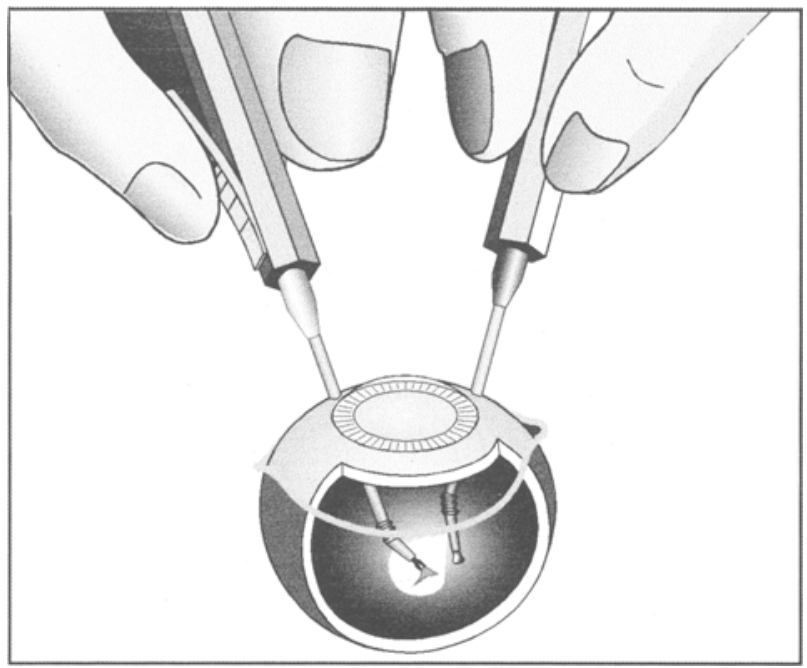

Fig. 4. Overview of the prototype. The angle of the end-neck is adjusted after the first insertion, then fixed by the lock mechanism while the surgeon works upon the tissue. 


\subsection{Design concepts of prototype}

The basic features of developed Micro Active Forceps (MAF) may be summarized as follows:

1) Active joint with lock mechanism to make the end-effector approach the diseased part flexibly and address the upper part of eyeball from the inside,

2) Micro gripper to grasp fine tissue on the retina,

3) Built-in optical fiber scope with light guide to obtain lateral images during operation,

4) One hand operation for all tasks is possible,

5) Solo surgery is possible, because a surgeon can undertake two-arm operation (bimanual) using two micro active forceps.

Fig. 4 shows the overview of the prototype, and the basic design is described as Fig. 8 .

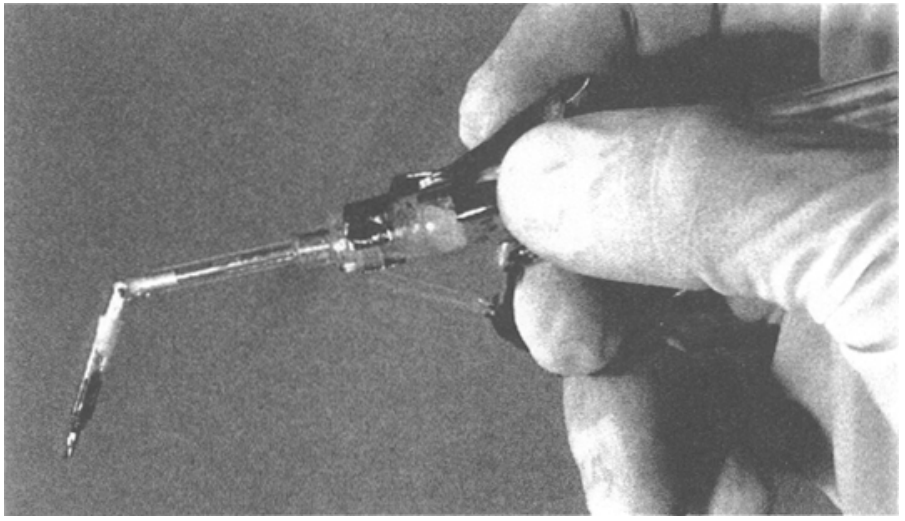

Fig. 5. Maneuver of MAF-II. The bending angle of the end-neck is adjusted by the middle finger and is held by the thumb via the lock mechanism. Then the gripper is worked stably by a forefinger. Each finger's force is transmitted via the nylon wire.

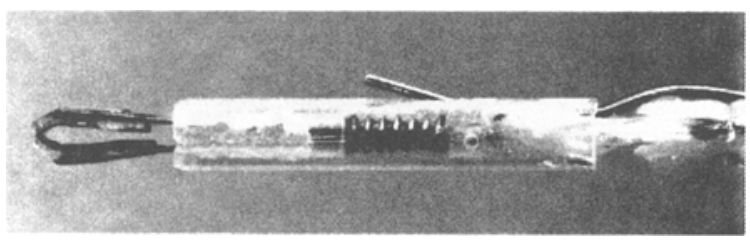

Fig. 6. A close-up view of the end-neck. The guide tube of the micro fiberscope is equipped obliquely. The lateral images of another instrument's tip and retina are obtained through the optical fiber. 
Firstly, a mechanism verification model, named MAF-II, was made by hand (Fig. 5 and 6). The shaft diameter is $3 \mathrm{~mm}$ and micro optical fiberscope (diameter is $0.4 \mathrm{~mm}$ with 2000 pixels) is set at 30 degrees on the tip of the forceps to obtain lateral images of the retina. The feasibility and maneuverability of the MAF-II was verified experimentally, which is explained in a later section.

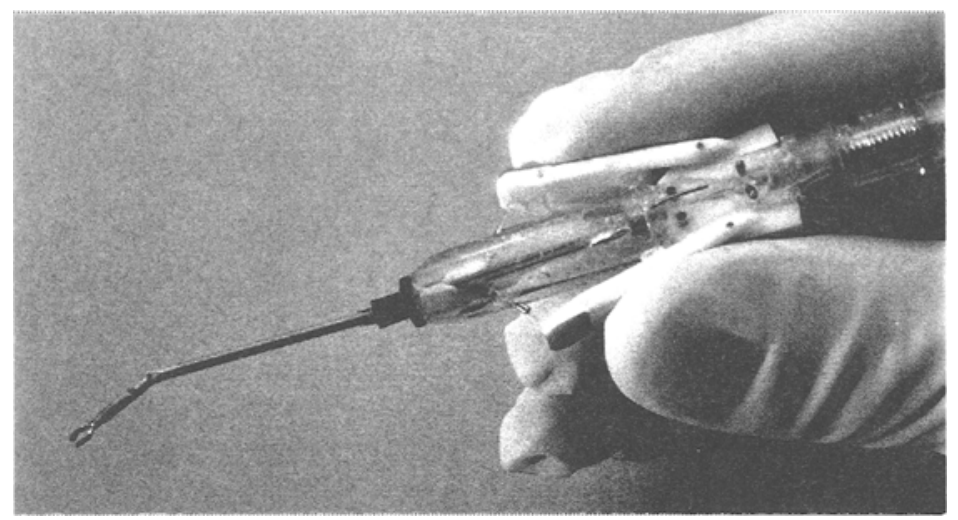

Fig. 7. Maneuver of MAF-III. Both the gripper and the bending angle are controlled simply by two fingers. The shape of the prototype comes to be similar to that of conventional forceps.

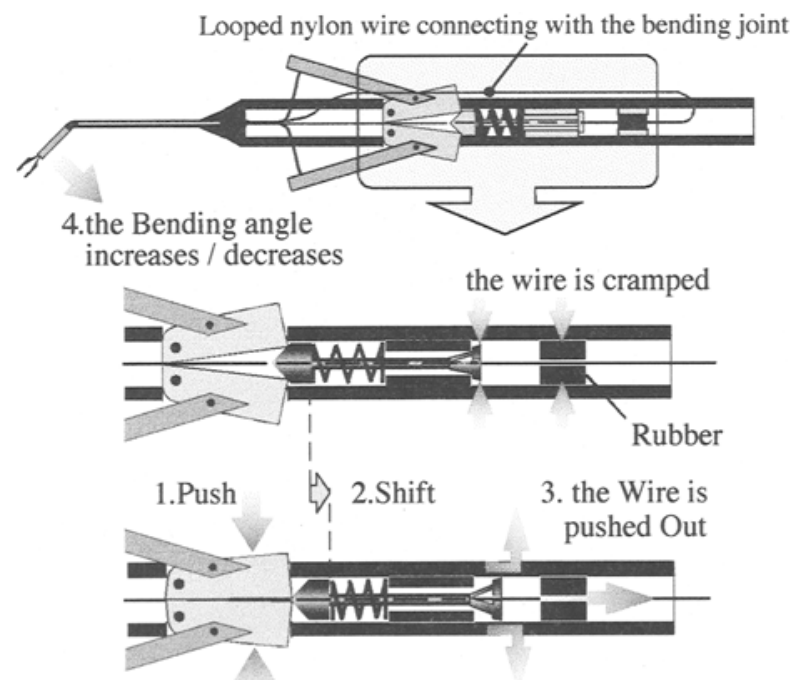

Fig. 8. Basic design of MAF. To earn the bending angle of the joint, the cramped point of looped wire is pushed out by knocking the knob just like the lead of a mechanical pencil. 
As the next step of trial production, the MAF-III was made, in which the diameter of the shaft has been thin downed (Fig. 7). $1.4 \mathrm{~mm}$ of ciameter is close to the size of today's micro instruments. Further miniaturization of the diameter in the next step is possible by using state of the art micro fabrication technology. An active bending joint and micro gripper can be operated by one hand via thin micro wire running through the body of MAF. The bending angle of the active joint and the micro gripper are separately maneuvered by a forefinger and a thumb respectively.

\subsection{Mechanical design of the end-neck}

In this section, let us examine the required kinematic conditions of the end-neck of MAF. There are two important factors that characterize motions of the tip against the surgical point: length of the end-neck and bending angle of the active joint. Both design parameters must be optimized So we started by determining the bending range, and also examined the length of the end-neck.

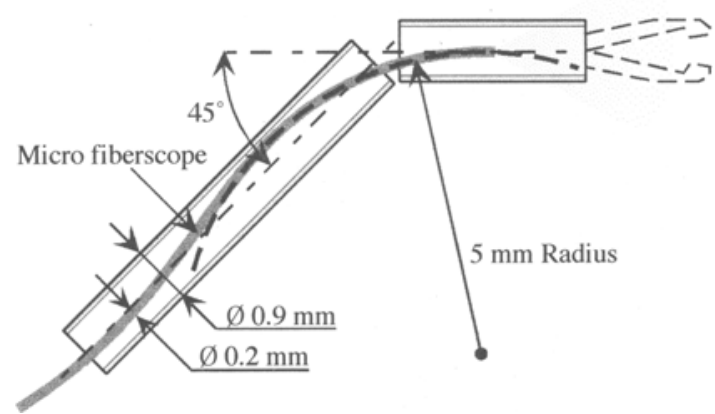

Fig. 9. The bending range of the active joint. Maximum bending angle ( 45 degrees) depends on the permitted bending radius of a fiberscope runs inside the stem.

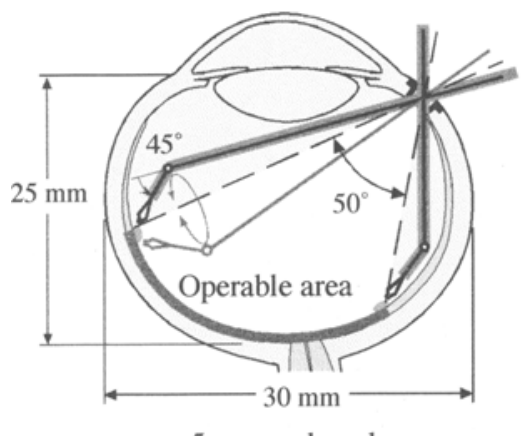

$5 \mathrm{~mm}$ end-neck

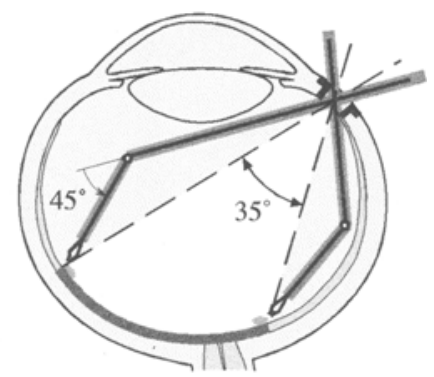

$10 \mathrm{~mm}$ end-neck

Fig. 10. Operable area of the retina under conditions of two different length of the endneck, $5 \mathrm{~mm}$ and $10 \mathrm{~mm}$. 


\subsubsection{Determination of bending angle}

The bending angle of an active joint mostly depends on the permissible bending radius (PBR) of the built-in fiberscope. The diameter of the fiberscope is $0.2 \mathrm{~mm}$ and PBR is $5 \mathrm{~mm}$, and the inside diameter of the neck is $0.6 \mathrm{~mm}$.

Considering sizes above as Fig. 9, the bending angle was assigned to the following range: $0^{\circ} \leq \theta \leq 45^{\circ}$.

\subsubsection{Length of the end-neck}

Next, the optimum length $L$ of the end-neck was examined. It is considered to be in the sphere of $5 \mathrm{~mm} \leq \mathrm{L} \leq 10 \mathrm{~mm}$. Where $5 \mathrm{~mm}$ is due to the mechanical limitation, while $10 \mathrm{~mm}$ is due to the size of an eyeball (25 mm depth). Compared to the conventional forceps, MAF with $10 \mathrm{~mm}$ or more longer end-neck have no effect on enlargement of the operable area of the retina.

Fig. 10 shows the comparison with operable ranges of the retina under conditions of 45 degrees of bending angle. It is obvious that the operable area by $5 \mathrm{~mm}$ end-neck is larger than that by $100 \mathrm{~mm}$ end-neck. So the optimum length of the end-neck should be fixed at $5 \mathrm{~mm}$.

\subsection{Total system}

Fig. 11 shows the total micro surgical system. The eye surgeon can easily grasp and pick up fine tissues on the retina by using two micro active forceps. A superimposed lateral microscopic image is provided from optical fiberscope along with the top view image by binocular microscope. The three-dimensional relation of positions between the tip of MAF and the retina can be clearly understood.

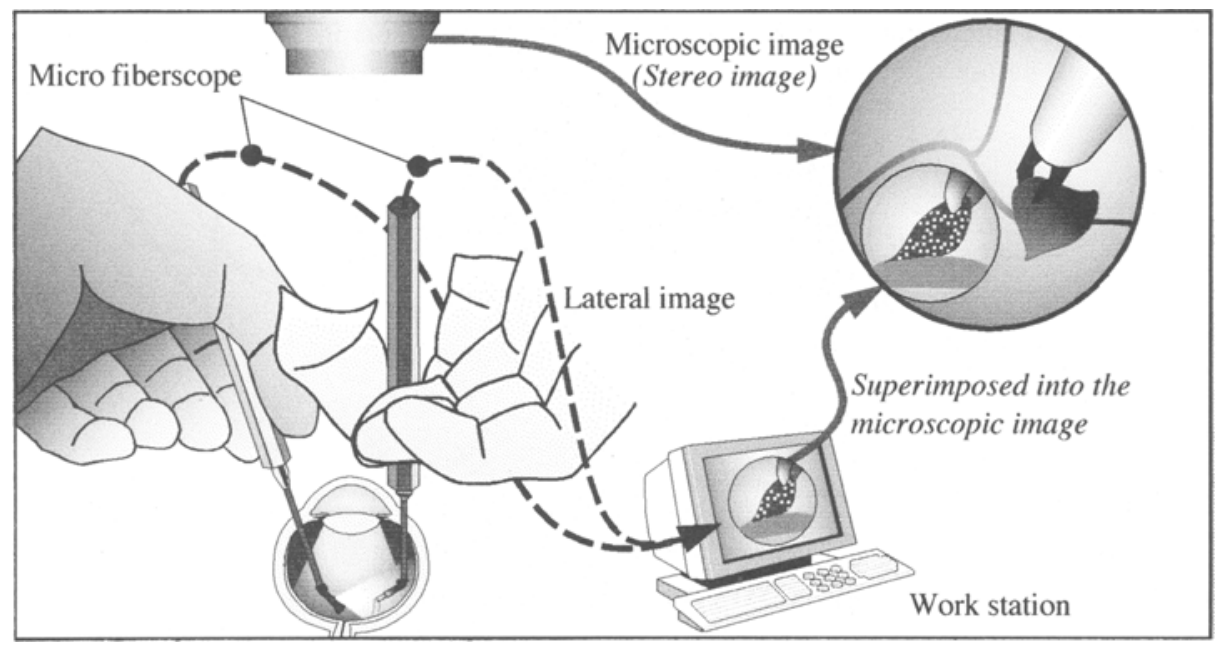

Fig. 11. Proposed total system of retina surgery using micro active forceps with superimposed lateral image. 


\section{Experimental Evaluation}

A preliminary experiment using pig's eyeball was conducted (Fig. 12). The micro active forceps was inserted through the cornea.

There were two main aims as follows:

1) Confirmation of maneuverability of the prototype,

2) Validity of lateral monitoring via fiberscope.

The blood capillary on the retina was grasped softly by the micro gripper. Fig. 13 shows the real lateral image via an optical fiberscope. The distance between the micro gripper and a thin blood capillary can be readily sensed. A surgeon can see both the top view and the lateral view as in Fig. 14.

One hand operation without an assistant was also verified successfully.

Feasibility and maneuverability were verified by eye micro surgeon. The optimization and improvement of the instrument are underway.

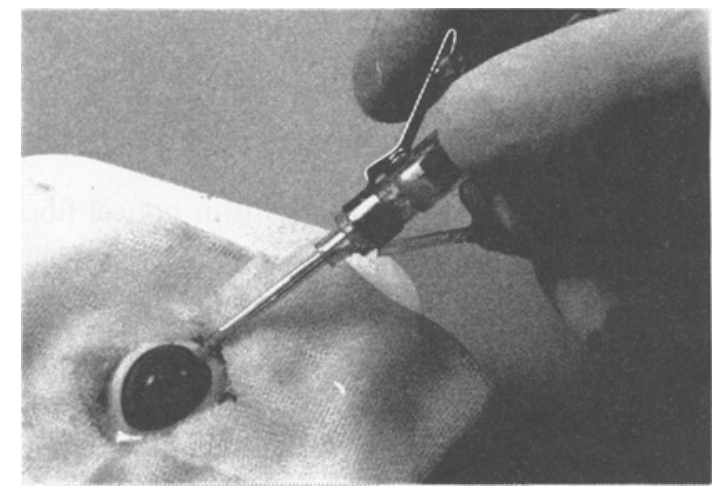

Fig. 1 2. Maneuverability test using a pig's eyeball.

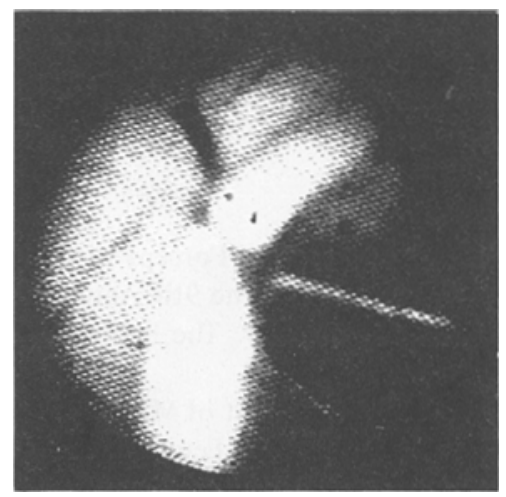

Fig. 13. Lateral image via optical fiber scope. Micro gripper is handing blood capillary on the retina. 


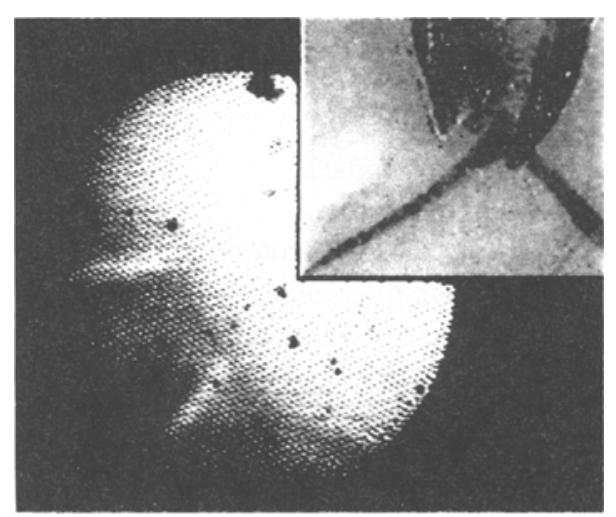

Fig. 14. The microscopic top view image is superimposed on the lateral view image via micro fiber scope.

\section{Conclusions}

A micro active forceps with micro gripper and built-in optical fiber scope was developed for eye microsurgery on the retina. In adkition, a total system, including a new display system able to see the lateral inside view of the tip, was proposed and tested. The maneuverability and feasibility of the prototype was verified experimentally. Onehand operation was also demonstrated.

\section{Acknowledgments}

We would like to acknowledge for presenting optical fiber at Mr. Utsumi of Mitsubishi Cable Co. Ltd. and Mr. Narita of Nippon Cable co. Ltd.

\section{References}

1. Ikuta, K., Kato, T., Nagata, S.: Micro Active Forceps with Optical Fiber Scope for IntraOcular Microsurgery. In: Proceedings of The 9th Annual International Workshop on MEMS '96. IEEE Catalog No. 96CH35856. The Institute of Electrical and Electronics Engineers, Inc. (1996) $456-461$

2. Ikuta, K., Kato, T., Nagata, S.: Development of Micro Active Forceps with Built-in Fiber Scope for Intra-Ocular Microsurgery. In: Lemke, H. U., Vannier, M.W., Inamura, K. (eds.): Proceedings of the 11 th International Symposium and Exhibition on CAR '97. International Congress Series, No. 1134. Elsevier Science B. V., Amsterdam Lausanne New York Oxford Shannon Tokyo (1997) 914 - 919

3. Nagata, M., Ogino, N., Okinami, S., Matsumura, M. (eds.): Ophthalmic Microsurgery 3rd edition. Medical Information Express, Tokyo Osaka (1993) 